\title{
Verticillium longisporum infection induces organ-specific glucosinolate degradation in Arabidopsis thaliana
}

\author{
Katja Witzel*, Franziska S. Hanschent, Rebecca Klopsch, Silke Ruppel, \\ Monika Schreiner and Rita Grosch
}

Leibniz Institute of Vegetable and Ornamental Crops, Grossbeeren, Germany

\section{OPEN ACCESS}

Edited by:

Masakazu Hara

Shizuoka University, Japan

Reviewed by:

Uener Kolukisaoglu,

University of Tuebingen, Germany

Xia Wu,

University of Washington, USA

*Correspondence:

Katja Witzel,

Leibniz Institute of Vegetable and Ornamental Crops,

Theodor-Echtermeyer-Weg 1 ,

14979 Grossbeeren, Germany

witzel@igzev.de

tThese authors have contributed equally to this work

Specialty section:

This article was submitted to

Plant Physiology,

a section of the journal

Frontiers in Plant Science

Received: 02 April 2015

Accepted: 25 June 2015

Published: 10 July 2015

Citation:

Witzel K, Hanschen FS, Klopsch R,

Ruppel S, Schreiner M and Grosch $R$

(2015) Verticillium longisporum

infection induces organ-specific

glucosinolate degradation

in Arabidopsis thaliana.

Front. Plant Sci. 6:508.

doi: $10.3389 /$ fpls.2015.00508
The species Verticillium represents a group of highly destructive fungal pathogens, responsible for vascular wilt in a number of crops. The host response to infection by Verticillium longisporum at the level of secondary plant metabolites has not been well explored. Natural variation in the glucosinolate (GLS) composition of four Arabidopsis thaliana accessions was characterized: the accessions Bur-O and Hi-O accumulated alkenyl GLS, while 3-hydroxypropyl GLS predominated in $\mathrm{Kn}-0$ and Ler-O. With respect to GLS degradation products, $\mathrm{Hi}-\mathrm{O}$ and $\mathrm{Kn}-0$ generated mainly isothiocyanates, whereas Bur-0 released epithionitriles and Ler-0 nitriles. An analysis of the effect on the composition of both GLS and its breakdown products in the leaf and root following the plants' exposure to $V$. longisporum revealed a number of organ- and accession-specific alterations. In the less disease susceptible accessions Bur-0 and Ler-0, colonization depressed the accumulation of GLS in the rosette leaves but accentuated it in the roots. In contrast, in the root, the level of GLS breakdown products in three of the four accessions fell, suggestive of their conjugation or binding to a fungal target molecule(s). The plant-pathogen interaction influenced both the organ- and accession-specific formation of GLS degradation products.

Keywords: glucosinolate breakdown products, natural variation, plant root, plant secondary metabolites, vascular pathogen

Abbreviations: 3But-CN, 4-pentenenitrile; 3But-GLS, 3-butenyl GLS; 3But-ITC, 3-butenyl ITC; CETB, 1 cyano-3,4-epithiobutane; CETP, 1-cyano-2,3-epithiopropane; CETPent, 1-cyano-4,5-epithiopentane; CHETB, 1-cyano-2-hydroxy-3,4-epithiobutane; CN, cyanide; GLS, glucosinolate; I3M-GLS, indole-3-ylmethyl GLS; IAN, 3-indoleacetonitrile; IST, internal standard; ITC, isothiocyanate; 4MO-IAN, 4-methoxy-3-indoleacetonitrile; 1MOI3M-GLS, 1-methoxyindole-3-ylmethyl GLS; 4MOI3M-GLS, 4-methoxyindole-3-ylmethyl GLS; 3MSOP-GLS, 3-(methylsulfinyl)propyl GLS; 4MSOB-GLS, 4-(methylsulfinyl)butyl GLS; 7MSOH-GLS, 7-(methylsulfinyl)heptyl GLS; 5MSOP-ITC, 5-(methylsulfinyl)pentylITC; 8MSOO-CN, 9-(methylsulfinyl)nonylnitrile; 8MSOO-GLS, 8(methylsulfinyl)octyl GLS; 3MTP-CN, 4-(methylthio)butylnitrile; 3MTP-GLS, 3-(methylthio)propyl GLS; 4MTB-GLS, 4-(methylthio)butyl GLS; 4MTB-ITC, 4-(methylthio)butyl ITC; 6MTH-CN, 7-(methylthio)heptylnitrile; 7MTH-CN, 8-(methylthio)octylnitrile; 6MTH-ITC, 6-(methylthio)hexyl ITC; 7MTH-ITC, 7-(methylthio)heptyl ITC; 7MTH-GLS, 7-(methylthio)heptyl GLS; 8MTO-CN, 9-(methylthio)nonylnitrile; 8MTO-GLS, 8-(methylthio)octyl GLS; 8MTO-ITC, 8-(methylthio)octyl ITC; (R)2OH3But-GLS, (R)-2-OH-3-butenyl GLS; (S)2OH3But-GLS, (S)-2-OH-3-butenyl GLS; 4OHI3M-GLS, 4-hydroxyindole-3-ylmethyl GLS; 3OHP-GLS, 3-hydroxypropyl GLS; 4Pent-GLS, 4-pentenyl GLS; 4Pent-ITC, 4-pentenyl ITC; 2PE-CN, 3-phenylpropanenitrile; 2Prop-CN, 3-butenenitrile; 2Prop-GLS, 2-propenyl GLS; 2Prop-ITC, 2-propenyl ITC; 2OH3But-CN, 3-hydroxypentenenitrile; 3OHP-GLS, 3-hydroxypropyl GLS; 3OHP-CN, 4-hydroxybutylnitrile; 3OHP-ITC, 3-hydroxypropyl ITC; n.d., not detected. To facilitate understanding from which GLS the breakdown products derived, nitriles were abbreviated using the cyanide $(\mathrm{CN})$ name. 


\section{Introduction}

A characteristic feature of Brassicaceous plants is the presence of glucosinolates (GLS), a group of sulfur-containing secondary metabolites which contribute to the plant's defense against a range of biotic stresses (Agerbirk et al., 2009; Textor and Gershenzon, 2009). While GLS content is typically up-regulated by pathogen or pest attack, other forms of stress, notably UVB radiation (Mewis et al., 2012b) and drought (Mewis et al., 2012a) can also induce their accumulation. The 130 known GLS variants have been classified, according to the nature of the side chain present, into the aliphatic, aromatic and indole GLS (Agerbirk and Olsen, 2012). GLS are found in the vacuole; when the cell is disrupted, they interact with myrosinase to form either ITCs or nitriles. If the epithiospecifier protein (ESP) is present, the degradation process of alkenyl GLS generates an epithionitrile. The ESP protein has been also identified to favor the formation of nitriles from other (non-alkenyl) GLS (Wittstock and Burow, 2010; Kissen et al., 2012). In Arabidopsis thaliana, a number of other modifiers is known (Zhang et al., 2006; Burow et al., 2009; Kissen and Bones, 2009). Isothiocyanates (ITCs) act as an effective deterrent against many pathogens, including fungi, bacteria and even insects (Shofran et al., 1998; van Ommen Kloeke et al., 2012; Witzel et al., 2013). Nitriles and epithionitriles are generally less bioactive (Shofran et al., 1998; Matusheski and Jeffery, 2001; Wittstock et al., 2003).

A number of fungi belonging to the species Verticillium induce plants to wilt when they invade the vascular system. They can be responsible for significant losses in both crop yield and quality. The species complex has a broad host range, infecting lettuce, olive, cotton, eggplant and tomato, among others (Daayf et al., 1995; Tsror et al., 2001; Dervis et al., 2009; Atallah et al., 2011; Jimenez-Diaz et al., 2012). These hemibiotrophic species colonize the plant root surface in response to the presence of specific root exudates, and after penetrating the cortex and endodermis, spread systemically via the xylem in the form of conidia (Fradin and Thomma, 2006; Zhou et al., 2006). Heavy colonization of the xylem can obstruct the transpiration stream, forcing the plant to form new xylem tissue (Reusche et al., 2012). One of the known host responses to Verticillium infection is an adjustment in the level and identity of secondary metabolites (Floerl et al., 2012; Iven et al., 2012; Obermeier et al., 2013; König et al., 2014). Linking diversity in secondary metabolite profiles (especially GLS) to the host/pathogen interaction is a science still in its infancy (Moore et al., 2014). In particular, little effort has been made to factor in crosstalk between the above and below ground parts of the plant in the context of defense against soil-borne pathogens.

A previous screen characterized GLS profiles of 19 A. thaliana accessions and related the growth-suppressive effect of volatile GLS breakdown products on Verticillium longisporum growth to the abundance of 2-propenyl ITC (2Prop-ITC) in a biofumigation assay (Witzel et al., 2013). Here, we extend this study to investigate the in planta influence of fungal colonization on GLS and their bioactive breakdown products.
The effect on these traits of colonization by $V$. longisporum has been investigated, by comparing the performance of four accessions chosen to contrast not just with respect to their GLS (alkenyl GLS: Bur-0, Hi-0; hydroxyalkenyl GLS: Kn0 , Ler-0) and GLS breakdown product profiles, but also in the extent of their susceptibility to $V$. longisporum infection. We hypothesize that metabolic fingerprinting of a group of important defense compounds in A. thaliana accessions with quantitative variation in fungal tolerance provides new clues to understand tissue-specific GLS partitioning in response to pathogens.

\section{Materials and Methods}

\section{Chemicals}

2Prop-ITC, $(\geq 99 \%)$, benzonitrile ( $\geq 99.9 \%)$, 3-butenenitrile (2Prop-CN, $\geq 98 \%$ ), 4-pentenenitrile (3But-CN, $\geq 97 \%$ ), 3phenylpropanenitrile (2PE-CN, $\geq 99 \%)$ and sucrose-sodium nitrate media were purchased from Sigma-Aldrich Chemie $\mathrm{GmbH}$, Steinheim, Germany; IAN, ( $\geq 98 \%$ ) from Acros Organics (Fischer Scientific GmbH, Schwerte, Germany); 3-butenyl ITC (3But-ITC, $\geq 95 \%$ ) and 4-pentenyl ITC (4Pent-ITC, $\geq 95 \%$ ) were purchased from TCI Deutschland GmbH, Eschborn, Germany; 3-hydroxypropionitrile was purchased from Thermo Fischer Scientific, Erembodegem, Belgium; 4-(methylthio)butyl ITC (4MTB-ITC, $\geq 98 \%$ ) was purchased from Santa Cruz Biotechnology, Heidelberg, Germany; 5-(methylsulfinyl)pentyl ITC (5MSOP-ITC) was purchased from Enzo Life Sciences GmbH, Lörrach, Germany; 1-cyano-2,3-epithiopropane (CETP), $(\geq 95 \%)$ was purchased from Taros Chemicals GmbH Co. KG, Dortmund, Germany; 4-hydroxybenzyl GLS ( $\geq 97 \%$ ), methylene chloride (GC Ultra Grade), Tris, EDTA, NaCl, CTAB, chloroform/isoamylalcohol (24:1), $\beta$-mercaptoethanol and phenol/chloroform/isoamylalcohol (25:24:1) from Carl Roth GmbH, Karlsruhe, Germany; acetonitrile (Ultra Gradient HPLC grade) was purchased from J.T. Baker, Deventer, The Netherlands and $\mathrm{NaSO}_{4}(\geq 99 \%)$ and methanol (>99.9) were purchased from VWR International GmbH, Darmstadt, Germany. Potato dextrose agar was purchased from Merck, Darmstadt, Germany. All solvents were of LC or GC-MS grade.

\section{Plants, Growing Conditions, and Fungal Inoculation}

Seed of the A. thaliana accessions Bur- 0 , Hi- $0, \mathrm{Kn}-0$ and Ler-0 (kindly provided by L. Westphal, Leibniz Institute of Plant Biochemistry, Germany), selected on the basis of their contrasting GLS profiles (Witzel et al., 2013), were germinated in soil and transplanted into quartz sand after 2 weeks. The plants were fed with a liquid nutrient formulation (Gibeaut et al., 1997) and exposed to an $8 \mathrm{~h}$ photoperiod provided by $300 \mu \mathrm{mol} \mathrm{m} \mathrm{m}^{-2} \mathrm{~s}^{-1}$ light at $20^{\circ} \mathrm{C}$ (light period) $/ 18^{\circ} \mathrm{C}$ (dark period).

V. longisporum isolate 43-3 (Zeise and von Tiedemann, 2002, kindly provided by A. von Tiedemann, Georg-August-University, Germany) was maintained on potato dextrose agar plates, and 
mycelial suspensions were prepared as described by Witzel et al. (2013). Briefly, a flask containing $100 \mathrm{~mL}$ SSN medium was inoculated with six $5 \mathrm{~mm}$ diameter agar disks cut from the margin of an actively growing $V$. longisporum culture and incubated for 1 week. A further $200 \mathrm{~mL}$ SSN medium was added, and the culture maintained for two additional weeks. After mechanical blending, the fragmented mycelial suspensions were centrifuged and the pellets rinsed twice by resuspension in sterile tap water. Conidia were counted in a Thoma chamber to allow the concentration to be adjusted to $10^{6} \mathrm{~mL}^{-1}$. A $10 \mathrm{~mL}$ aliquot (or $10 \mathrm{~mL}$ water for mock inoculations) of the conidial suspension was poured over the surface of a pot containing the 2 weeks-old $A$. thaliana seedlings, which were then grown on for a further 4 weeks. Rosette leaves and roots were harvested, snap-frozen and ground to a powder, which was stored at $-80^{\circ} \mathrm{C}$ until required for the extraction of DNA, proteins and metabolites.

Plant growth measurements were taken of the fresh weight of root and rosette of 20 plants of all four A. thaliana accessions, grown under control conditions or inoculated with $V$. longisporum, in three independent experiments. The relative growth rate was determined from the ratio between growth of control and inoculated plants. Analysis for statistical significance was done using Student's $t$-test implemented in SigmaPlot 12.3 software (SPSS Inc., Chicago, IL, USA).

\section{DNA Isolation and Quantitative Real-Time PCR (qPCR)}

Genomic DNA was isolated using the CTAB extraction method (Doyle and Doyle, 1990; Tinker et al., 1993). Aliquots of $200 \mathrm{mg}$ powdered root material were homogenized in $500 \mu \mathrm{L}$ CTAB [100 mM Tris-HCl pH 8, $1.4 \mathrm{M} \mathrm{NaCl}, 20 \mathrm{mM}$ EDTA, 2\% $(\mathrm{w} / \mathrm{v}) \mathrm{CTAB}, 0.2 \%(\mathrm{v} / \mathrm{v}) \beta$-mercaptoethanol], then extracted by the addition of $200 \mu \mathrm{L}$ chloroform/isoamylalcohol (24:1), After centrifugation, the DNA pellet was dissolved in $200 \mu \mathrm{L}$ nucleasefree water and treated with RNase A $\left(15 \mu \mathrm{L}\right.$ of $50 \mu \mathrm{g} \mathrm{mL}^{-1}$ RNase). Following a phenol/chloroform/isoamylalcohol (25:24:1) extraction, the DNA was precipitated in acidic ethanol (Gebhardt et al., 1989) and dissolved in $10 \mathrm{mM}$ Tris-HCl/1 mM EDTA ( $\mathrm{pH}$ 8). DNA concentration was derived via a Nano Drop ND-1000 spectrophotometer (Peqlab, Erlangen, Germany). The abundance of fungal DNA present in the DNA sample was estimated by a qPCR assay based on the primer pair VDS1/2 (5'-CAC ATT CAG TTC AGG AGA CGG A/5' -CCT TCT ACT GGA GTA TTT CGG) which amplifies a $521 \mathrm{nt}$ stretch of $V$. longisporum DNA (Li et al., 1999). Detection of $V$. longisporum was performed as described in Witzel et al. (2013).

\section{Analysis of GLS and their Breakdown Products}

The GLS composition of the A. thaliana leaf and root samples was determined as desulfo-GLS, using a slightly modified form of the Wiesner et al. (2013b) method. The modifications were as follows: the extraction was based on $10 \mathrm{mg}$ of lyophilized plant material, and the internal standard was a $0.05 \mu \mathrm{mol}$ aliquot of 4-hydroxybenzyl GLS. The various desulfo-GLS were separated by a UHPLC-DAD device (UHPLC Agilent 1290 Infinity System, Agilent Technologies, Böblingen, Germany) equipped with a Poroshell 120 EC-C18 column of dimension $100 \mathrm{~mm} \times 2.1 \mathrm{~mm}$ containing particles of size $2.7 \mu \mathrm{m}$ (Agilent Technologies). The solvent gradient was formed by water (A) and $40 \%$ acetonitrile (B), starting at $0.5 \% \mathrm{~B}$ for $2 \mathrm{~min}$, rising to $49.5 \% \mathrm{~B}$ over the next $10 \mathrm{~min}$, then held for a further $2 \mathrm{~min}$, increased to $99.5 \% \mathrm{~B}$ over the course of $1 \mathrm{~min}$ and finally held for a final $2 \mathrm{~min}$. The flow rate was $0.4 \mathrm{~mL} \mathrm{~min}{ }^{-1}$ and the injection volume $5 \mu \mathrm{L}$. Desulfo-GLS were identified by comparing retention times and UV absorption spectra with those of known standards. Quantification was done at $229 \mathrm{~nm}$ via the internal standard (IST) 4-hydroxybenzyl GLS using the response factor (RF) of the GLS relative to 4-hydroxybenzyl GLS.

The quantification of GLS breakdown products was based on a GC-MS analysis, as described (Witzel et al., 2013; Piekarska et al., 2014), using an Agilent 7890A Series GC System (Agilent Technologies) equipped with an Agilent 7683 Series Autosampler, an Agilent 7683B Series Injector and an Agilent 5975C inert XL MSD. Analytes were separated using a SGE BP5MS column $30 \mathrm{~m} \times 0.25 \mathrm{~mm} \times 0.25 \mu \mathrm{M}$ (VWR International $\mathrm{GmbH}$, Darmstadt, Germany). The chosen instrument settings differed only slightly from those given by Piekarska et al. (2014): the temperature was set to $35^{\circ} \mathrm{C}$ for the initial $3 \mathrm{~min}$, then raised to $100^{\circ} \mathrm{C}$ at $9^{\circ} \mathrm{C} \mathrm{min}^{-1}$, where it was held for $3 \mathrm{~min}$; the rest of the protocol was identical to that given by Piekarska et al. (2014). Molecular species were identified by their mass spectrum and retention time in comparison with those of authenticated standards and with literature data (Kjaer, 1963; Spencer and Daxenbichler, 1980). Analyte content was calculated using benzonitrile as IST and the RFs of CETP $(\mathrm{RF}=1.66)$, 2Prop-ITC $(\mathrm{RF}=1.71)$, 2Prop-CN $(\mathrm{RF}=3.71)$, 3But-ITC $(\mathrm{RF}=1.28), 3$ But-CN $(\mathrm{RF}=2.61), 3$-hydroxypropionitrile $(\mathrm{RF}=7.12), 4 \mathrm{MTB}-\mathrm{ITC}(\mathrm{RF}=0.53), 5 \mathrm{MSOP}-\mathrm{ITC}(\mathrm{RF}=0.98)$, 3-hydroxypropionitrile ( $\mathrm{RF}=7.12), 2 \mathrm{PE}-\mathrm{CN}(\mathrm{RF}=0.54)$, and IAN $(\mathrm{RF}=0.35)$ relative to benzonitrile. For the commercially unavailable compounds, a response factor equal to that of the chemically most similar compound was assumed. Thus, other epithionitriles than CEPT itself were quantified at hand of the $\mathrm{RF}$ of CEPT; that of 3-hydroxypentene nitrile (2OH3But-CN) was based on that of 3But-CN (1.28), those of the breakdown products of the methylsulfinyl-alkyl GLS on that of 5MSOP-ITC (0.98), those derived from methylthioakyl GLS on that of 4MTBITC (0.53), the degradation products of 3-hydroxypropyl GLS on that of 3-hydroxypropionitrile (7.12), and that of 4-methoxy-3indoleacetonitrile (4MO-IAN) on that of IAN (0.35). The limit of detection ranged between $0.2 \mu \mathrm{M}(2 \mathrm{PE}-\mathrm{CN})$ and $10 \mu \mathrm{M}$ (3-hydroxypropionitrile).

Because of the extent of the inter-experiment variation for metabolite concentrations, quantitative changes in GLS and their breakdown products induced by fungal colonization were analyzed separately for each of the three experiments. Relative fold changes between the inoculated and the noninoculated plants were determined for each compound and the Student's $t$-test was applied to identify statistically significant differences in mean compound content, applying a threshold of $p<0.05$ (Figures 3 and 4). Absolute amounts of GLS and their respective breakdown products are presented in Supplementary 
Tables S1 and S2 as a mean of three independent experiments and the standard error.

Hierarchical clustering of profiles of GLS and GLS breakdown products was performed using MultiexperimentViewer $\mathrm{MeV}$ v4.7.4, based on Pearson correlation and average linkage clustering, on log2 transformed ratios between control and inoculated plants (Saeed et al., 2003).

To estimate the relation of total GLS breakdown products to the total amount of GLS, all values were converted to fresh weight basis. The recovery rate was determined as ratio between amount of total GLS breakdown products and amount of total GLS multiplied with $100 \%$ in three independent experiments. Analysis for statistical significance was done using Student's $t$-test implemented in SigmaPlot 12.3 software (SPSS Inc., Chicago, IL, USA).

\section{Western Blotting}

Proteins were extracted from the leaf and immunoblotted (based on $1 \mu \mathrm{g}$ protein) as described by Amme et al. (2005). The blots were probed with an antibody recognizing the A. thaliana ESP, an enzyme responsible for diverting GLS hydrolysis from the generation of ITCs to that of epithionitriles or nitriles (Kissen et al., 2012, kindly provided by Ralph Kissen). Signal intensity was quantified using Phoretix 1D v11.4 gel analysis software (TotalLab, Newcastle upon Tyne, UK).

\section{Results}

\section{Variation among the Four $A$. thaliana Accessions for Disease Susceptibility}

The impact of the $V$. longisporum inoculation was stronger on the roots than on the leaf (Figure 1). All four accessions experienced a decline in root biomass, with $\mathrm{Hi}-0$ and $\mathrm{Kn}-0$ being the most severely affected. Leaf biomass was also reduced in both Hi-0 and Kn-0, while leaf biomass accumulation in Bur0 and Ler- 0 was relatively unaffected by the fungus. The only visible above-ground disease symptom observed at the time of harvest was the reduced growth of $\mathrm{Hi}-0$ and $\mathrm{Kn}-0$, but no wilting. Based on the biomass response to inoculation, $\mathrm{Hi}-0$ and $\mathrm{Kn}-0$ were classified as susceptible, and Bur-0 and Ler-0 as tolerant to $V$. longisporum. When the quantity of fungal DNA present in the root DNA preparation was tested by qPCR, the extent of the fungal colonization in the Ler-0 and $\mathrm{Hi}-0$ roots was shown to be greater than in those of both Bur-0 and Kn-0 (Figure 2).

\section{The Effect of Fungal Colonization on GLS Profile}

In all, 17 distinct GLS compounds were detected in the leaf material and 16 in the root of plants not infected with

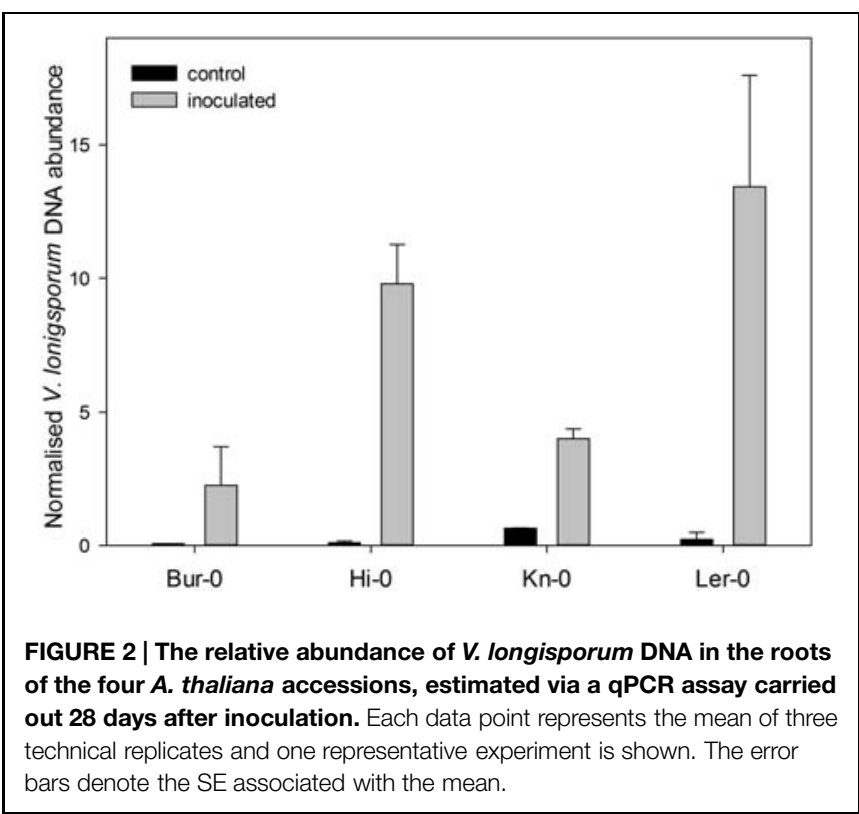

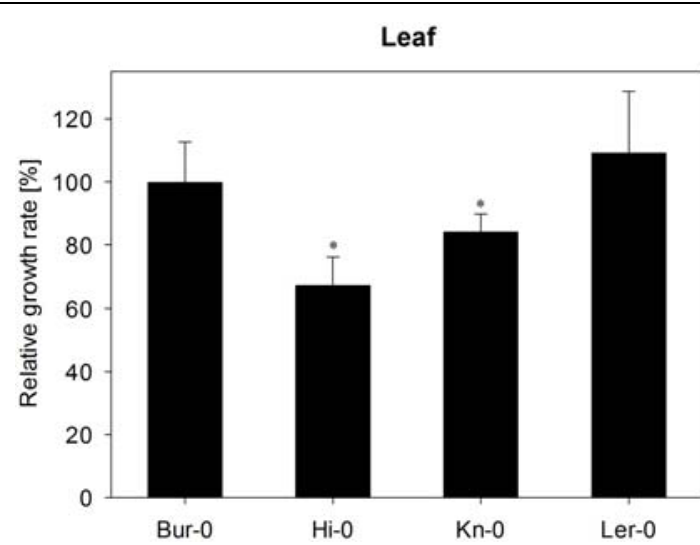

FIGURE 1 | The effect of Verticillium longisporum colonization on growth of Arabidopsis thaliana accessions Bur- $0, \mathrm{Hi}-0, \mathrm{Kn}-0$, and Ler-0. Each data point represents the mean of three independent experiments. Relative growth rates were calculated by comparing inoculated with non-inoculated

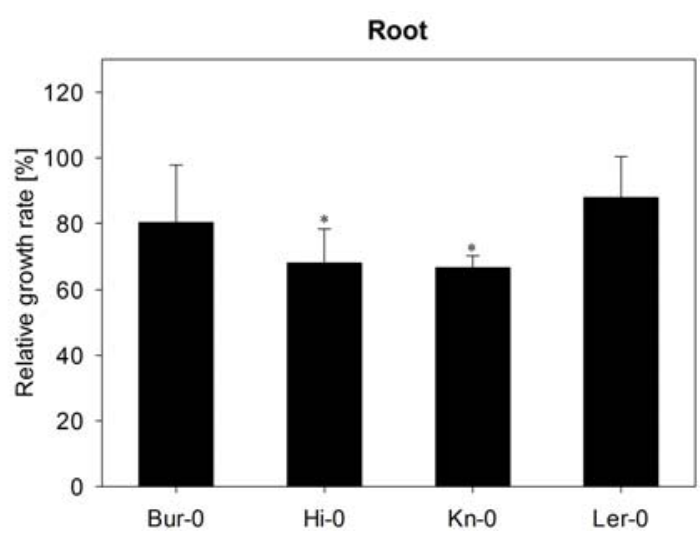

plants of each accession in turn on a fresh weight basis. The error bars denote the SE associated with the mean. Significant differences $(p<0.05)$ between inoculated and non-inoculated plants of the same accession are indicated by an asterisk. 


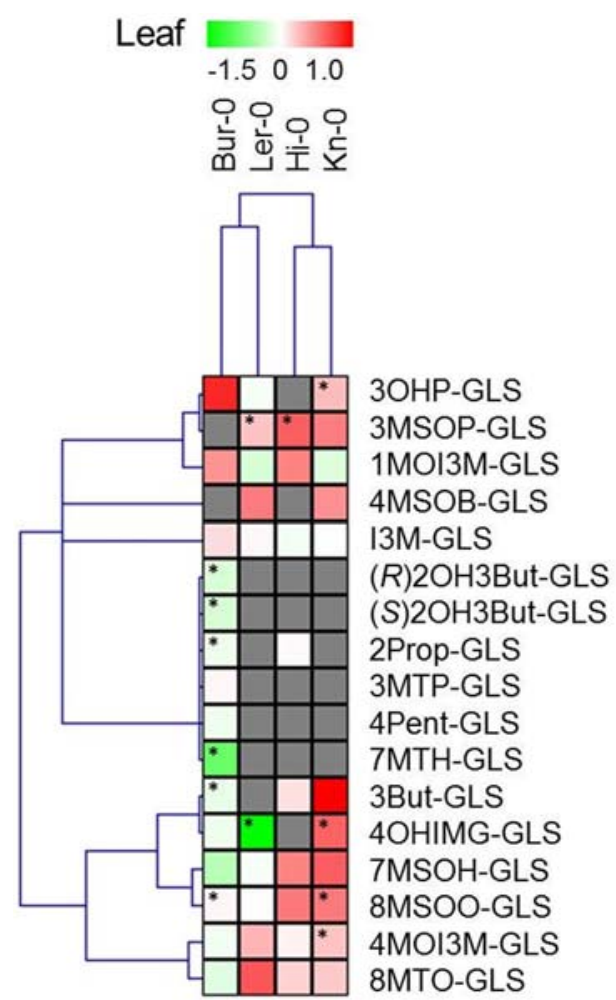

FIGURE 3|Changes in the GLS profile induced by colonization with V. Iongisporum. Each column represents a single accession and each row the fold change (inoculated/non-inoculated), calculated from three independent experiments and log2 transformed. Cells

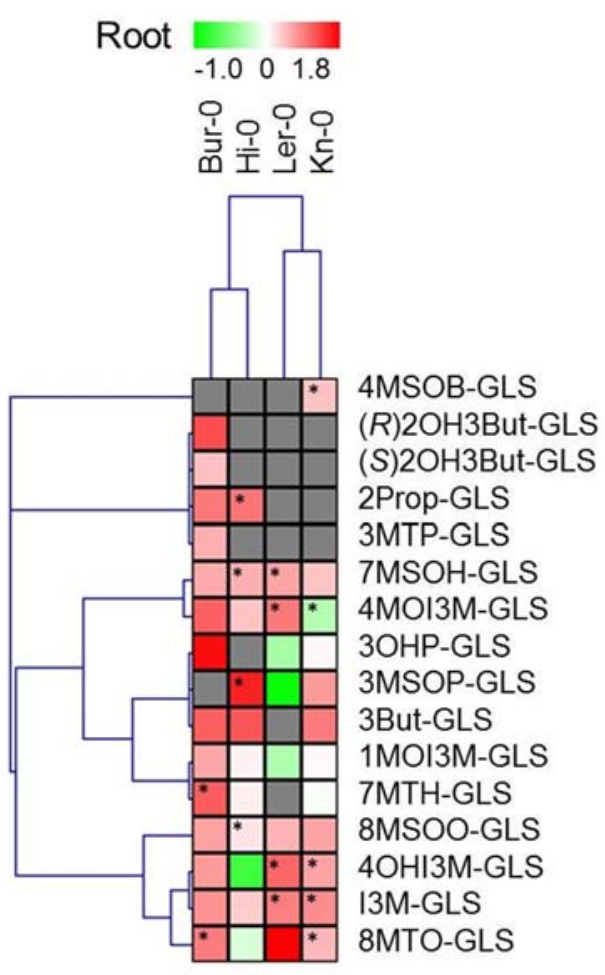

colored gray indicate the absence of the specific compound. Significant differences $(p<0.05)$ between inoculated and non-inoculated plants are indicated by an asterisk. Abbreviations are explained in the list of abbreviations.
$V$. longisporum. While the overall GLS contents of the two organ types were rather different, their composition was quite similar, and in accordance with earlier data (Witzel et al., 2013). A full tabulation of the various GLS compounds identified is presented as Supplementary Table S1; this includes those compounds which were inconsistently present and therefore not analyzed further. Overall, there were 15 compounds reliably present in Bur-0, nine in $\mathrm{Hi}-0$, eleven in $\mathrm{Kn}-0$ and ten in Ler-0. The predominant GLS in the Hi-0 and Bur-0 leaf were the alkenyls 2Prop-GLS and 3-butenyl GLS (3ButGLS) in Hi-0 and Bur-0, respectively, while the commonest GLS in the leaf of $\mathrm{Kn}-0$ and Ler-0 was 3-hydroxypropyl GLS (3OHP-GLS). The roots of Bur-0 contained substantial quantities of both 8-(methylsulfinyl)octyl GLS (8MSOO-GLS) and 1-methoxyindole-3-ylmethyl GLS (1MOI3M-GLS), while $\mathrm{Hi}-0$ roots in addition featured 2 Prop-GLS. In contrast, the GLS content of the $\mathrm{Kn}-0$ and Ler- 0 roots was dominated by 3 OHP-GLS.

In $V$. longisporum infected plants, the global GLS level was significantly higher than in the non-inoculated plants, especially in the root (Figure 3, Supplementary Table S1). For Bur-0, the root GLS content rose by $57 \%$, while that of the leaf fell by $7 \%$. Similarly, the root of Hi-0 accumulated 55\% more GLS than the non-infected root, while its leaf GLS content rose by $7 \%$. The effect of $V$. longisporum infection in $\mathrm{Kn}-0$ was an $11 \%$ rise in root GLS content and a $20 \%$ rise in leaf GLS content. Ler-0 was the accession least affected by the presence of the fungus: its root GLS content was enhanced by $3 \%$ while its leaf GLS content dropped by $6 \%$. With respect to GLS profile, in Bur0 roots, all of the GLS species were more abundant than in the non-infected roots, while in its leaf, the representation of ten GLS species was reduced, suggestive of GLS translocation toward the site of infection. The behavior of Ler- 0 was similar, featuring an increase in the presence of the indoles indole-3ylmethyl GLS (I3M-GLS) and 4-hydroxyindole-3-ylmethyl GLS (4OHI3M-GLS) and the sulfinylalkyls 8MSOO-GLS and 7(methylsulfinyl)heptyl GLS (7MSOH-GLS) in the infected root and a decrease in the leaf. In both $\mathrm{Hi}-\mathrm{O}$ and $\mathrm{Kn}-0$, the abundance of most of the GLS compounds rose in both the root and the leaf. The attempt to compare the GLS profiles of Bur-0 and Ler- 0 with those of $\mathrm{Hi}-0$ and $\mathrm{Kn}-0$ to identify which GLS species acted as a signature for $V$. longisporum tolerance was unsuccessful.

\section{The Effect of Fungal Colonization on the GLS Breakdown Product Profile}

The four A. thaliana accessions differed from one another not only with respect to their GLS profile, but also with respect to the spectrum of GLS breakdown products generated (Supplementary Table S2). Unsurprisingly, given that Bur-0 contained the greatest 


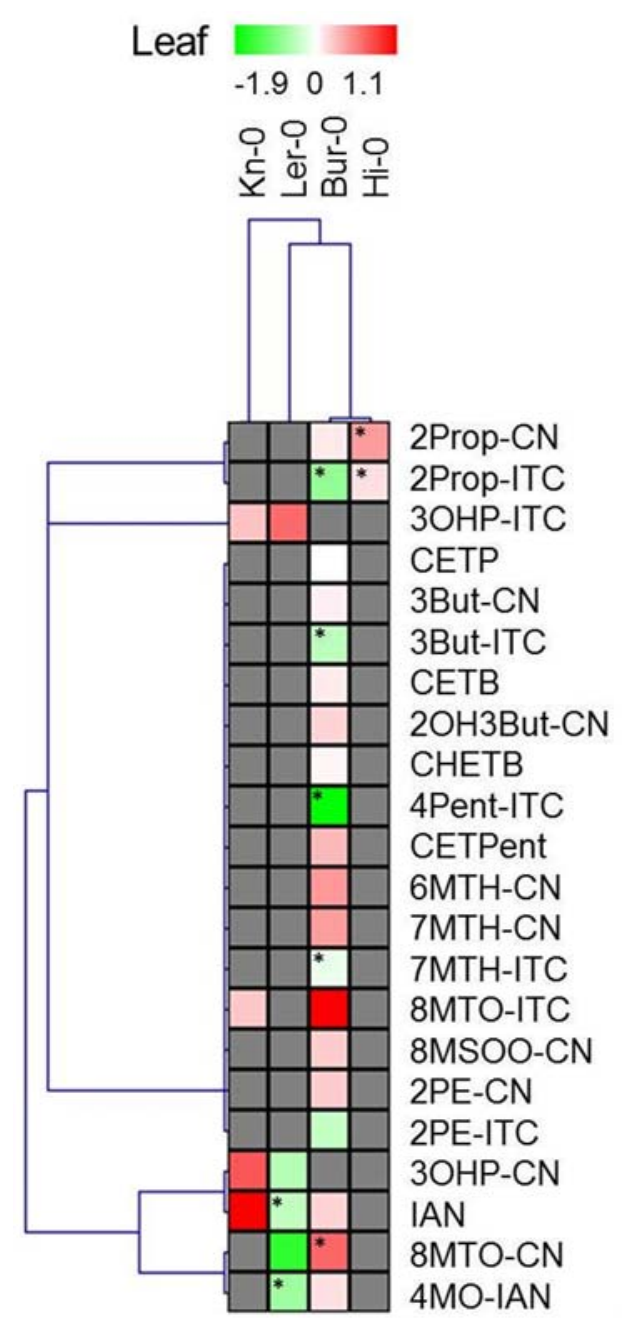

FIGURE 4 | Changes in the GLS breakdown product profile induced by colonization with $\boldsymbol{V}$. Iongisporum. Each column represents a single accession and each row the fold change (inoculated/non-inoculated) calculated from three independent experiments and log2 transformed. Cells

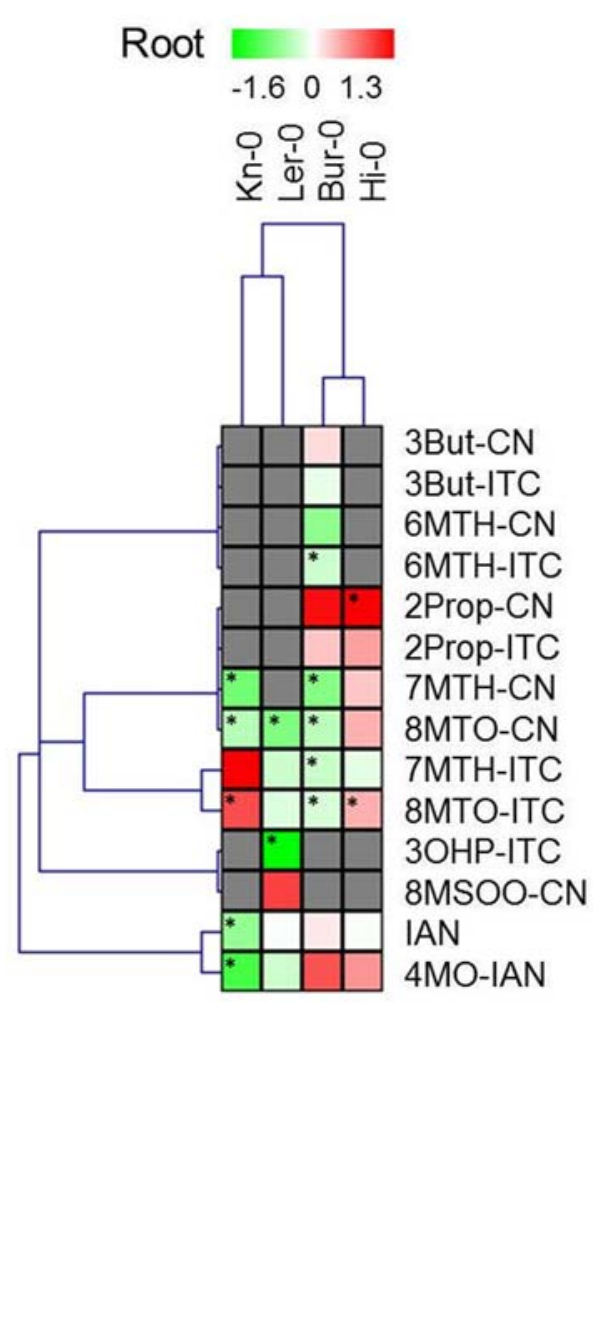

colored gray indicate the absence of the specific compound. Significant differences $(p<0.05)$ between inoculated and non-inoculated plants are indicated by an asterisk. Abbreviations are explained in the list of abbreviations. diversity of GLS species, its degradation product spectrum was also the most diverse, comprising 22 products; the number for Hi-0 was 14 , for Kn-0 eleven and for Ler- 0 ten. The dominant species in the Bur-0 leaf were the epithionitriles 1-cyano-3,4epithiobutane (CETB), derived from 3But-GLS, and 1-cyano-2,3epithiopropane (CETP), derived from 2Prop GLS due to high ESP protein abundance (Supplementary Figure S1). In the Ler-0 leaf, the most abundant products were 4-hydroxybutylnitrile (3OHP$\mathrm{CN}$ ) and the corresponding 3-hydroxypropyl ITC (3OHP-ITC). The Hi-0 leaf contained mainly 2Prop-ITC and lacked CEPT, while $90 \%$ of degradation products from 3OHP-GLS was the corresponding ITC in leaves of Kn-0. No ESP was detected in the root of both Bur-0 and Ler-0 (data not shown), and as result, the root GLS degradation profiles were rich in ITCs.

Infection with $V$. longisporum altered the spectrum of GLS degradation products in an accession-specific manner (Figure 4).
The Ler-0 leaf accumulated fewer nitriles, in accordance with its reduction in ESP abundance (Supplementary Figure S1). Although the abundance of ESP was reduced in the Bur-0 leaf, the formation of ITCs was less favored, while that of nitriles was enhanced. In both $\mathrm{Kn}-\mathrm{O}$ and $\mathrm{Hi}-\mathrm{O}$, the representation of both nitriles and ITCs increased. The quantity of GLS breakdown products in the leaf was enhanced by the fungal colonization in Bur-0, Hi-0 and Kn-0, but decreased in Ler- 0 . Three of the accessions (the exception was $\mathrm{Hi}-0$ ) responded to infection by accumulating fewer detectable degradation products in their root. In the case of $\mathrm{Hi}-0$, both ITC and nitrile levels were raised. Bur- 0 and Ler- 0 generated a reduced quantity of nitriles and of certain ITCs, while the Kn-0 profile involved an enhancement in ITC and a reduction in nitrile content. In Bur-0 and Ler-0, both the global GLS degradation product levels in the leaf and root were reduced upon inoculation: the reduction in the leaf was $7 \%$ in Bur-0 and $37 \%$ in Ler- 0 , while in the root the respective 
decreases were 17 and 30\%. However, in Kn-0, the leaf global GLS degradation product content was increased by $16 \%$, while that of the root fell by $21 \%$. Finally in $\mathrm{Hi}-0$, both the leaf and root content rose by, respectively, 11 and 53\%, as a result of $V$. longisporum spread. Relative to that of GLS, the concentration of breakdown products derived from GLS lay between $21 \%$ in the leaf of Ler-0 plants inoculated with $V$. longisporum and 104\% (noninoculated Bur-0 roots; Figure 5). In response to $V$. longisporum inoculation, a significant change in the relative concentration was only noted in the roots of Bur- 0 , where a decrease of $50 \%$ was estimated.

\section{Discussion}

The interaction between Verticillium and A. thaliana has been characterized at the level of host/pathogen signaling (Johansson et al., 2006; Pantelides et al., 2010; Tischner et al., 2010; Roos et al., 2014) and the genetic basis of host tolerance has been investigated (Veronese et al., 2003; Häffner et al., 2010, 2014). However, the influence of the plant's secondary metabolites on this interaction has not as yet been thoroughly investigated. The abundance of phenylpropanoids in the A. thaliana leaf tissue is enhanced when the plant is challenged by $V$. longisporum (König et al., 2014). A time-course study on the effect of the pathogen on tryptophan-derived secondary metabolites revealed an increase in root camalexin concentration and knock-out of biosynthetic genes increased susceptibility to $V$. longisporum (Iven et al., 2012). According to Witzel et al. (2013), certain GLS breakdown products are capable of inhibiting the growth of $V$. longisporum. The four selected $A$. thaliana accessions were chosen on the basis of their diversity in GLS and GLS breakdown product profiles, and were shown to vary with respect to their susceptibility to colonization by the pathogen (Figure 1). Although both Bur-0

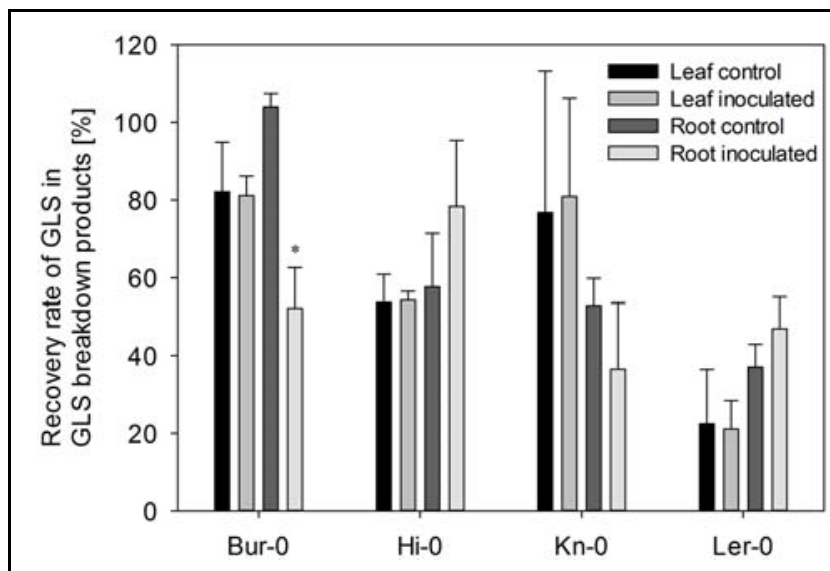

FIGURE 5 | The recovery rate of GLS in the form of breakdown products. Each data point represents the mean of three independent experiments. The recovery rate was calculated from the ratio between the total GLS content and total GLS breakdown product content for each experiment, accession and treatment separately. The error bars denote the SE associated with the mean. Significant differences $(p<0.05)$ between inoculated and non-inoculated plants of the same accession are indicated by an asterisk. and Ler-0 emerged as the more tolerant of the four, Häffner et al. (2010, 2014) considered Ler-0 as a sensitive genotype, despite using the same isolate as was employed here. This discrepancy is assumed to have arisen due to the difference in the inoculation method: in Häffner et al.'s $(2010,2014)$ experiments, plants were uprooted, the roots were trimmed and then exposed to a conidial suspension; whereas the present experiments were based on pouring a conidial suspension on the soil surface, then relying on the natural mode of fungal colonization of the host. Nevertheless, even though Ler- 0 was considered here as tolerant on the basis of its biomass accumulation capacity in the face of fungal infection, the abundance of the pathogen within the root of this accession was higher than in the other three accessions, confirming the observation of Häffner et al. (2010) that colonization rate does not correlate with disease severity.

\section{Verticillium Colonization Leads to the Accumulation of GLS in the Roots of Infected Plants}

GLS form a part of the plant's defense against biotic stress (Clay et al., 2009; Aires et al., 2011; Mewis et al., 2012a; Rohr et al., 2012), but few evidential data as yet support a role of GLS in the defense against soil-borne pathogens such as V. longisporum. Furthermore, interaction between above and below ground plant tissues in deterring soil-borne pathogens is scarcely investigated. Such systemic examinations should add to a better understanding of the biological basis for GLS variation between shoots and roots, as well as their role in restraining fungal pathogens. Colonization of the host by $V$. longisporum resulted in an increase in the root GLS content in all four accessions, while the GLS response in the leaf was inconsistent. The observed decrease in the case of Bur0 and Ler- 0 may reflect the transport of GLS from the shoot to the root (Madsen et al., 2014) or induced biosynthesis in roots. In Ler- 0 , the content of 4 OHI3M-GLS was significantly downgraded in the leaf, while it accumulated in the root, and the same applied to $7 \mathrm{MTH}-\mathrm{GLS}$ in Bur-0. It seems possible therefore that the translocation of GLS to the primary infection site contributes to the defense response of these accessions. Further testing of fungal colonization in A. thaliana genotypes that are either not able to accumulate GLS in their roots (Andersen et al., 2013) or that are deficient in specific GLS groups (Beekwilder et al., 2008) improve the understanding of the role of GLS in antagonize soil-borne pathogens.

\section{The Content of GLS Breakdown Products is Reduced by Verticillium Colonization}

The contribution of GLS to the host defense response has to date focused on the effect of intact GLS compounds (Beekwilder et al., 2008; Markovich et al., 2013), although it is well known that much of the bioactivity of GLS is associated with their breakdown products (reviewed by Hanschen et al., 2014). Analyses of the GLS breakdown product content in the tissues of stressed plants are scarce (Brader et al., 2006; Stotz et al., 2011). The enzymatic degradation of GLS generates a variety of molecules, the bioactivity of most of which still remains obscure. Some breakdown products, in particular the ITCs, have been shown to possess anti-bacterial and/or anti-fungal and/or anti-herbivore 
properties. The anti-carcinogenic effect of the ITCs is largely due to their ability to covalently bind to nucleophiles such as cysteine residues in tubulin, which inhibits, for example, tubulin polymerization, thus leading to cell growth inhibition and apoptosis induction (Mi et al., 2008). Treatment with a low concentration of ITCs can induce the expression of glutathione $S$-transferases, while at higher concentrations, the hydrogen peroxide generated leads to leaf bleaching (Hara et al., 2010). ITCs have also been implicated in the production of reactive oxygen species and the induction of stomatal closure (Khokon et al., 2011; Hossain et al., 2013), as well as the expression of heat-shock protein genes and an enhanced tolerance to high temperatures stress (Hara et al., 2013); these observations have suggested that ITCs do make a positive contribution to the plant's abiotic stress tolerance (Martinez-Ballesta et al., 2013). Treatment of $A$. thaliana leaves with 4-(methylsulfinyl)butyl ITC restricts the size of glutathione pool and induces a hypersensitive response (Andersson et al., 2015). Previously, 2Prop-ITC was found growth-inhibiting to $V$. longisporum in a plate assay using freeze-dried $A$. thaliana plant material (Witzel et al., 2013). However, GLS breakdown products differ between lyophilized and fresh plant material due to altered activity of modifying enzymes. In comparison to the earlier findings, formation of 2Prop-ITC was confirmed for $\mathrm{Hi}-0$ but declined for Bur-0. Main degradation product of 2Prop-GLS in leaves was the ITC (Hi-0) or epithionitrile (Bur-0), while CNs were formed in roots (Supplementary Table S2). As V. longisporum colonizes the plant via roots, 2 Prop- $\mathrm{CN}$ levels could be more relevant in deterring fungal spread than 2Prop-ITC, indicating that earlier findings might not account for the in planta interaction between host and pathogen. While ITCs predominate among the GLS degradation products, nitriles and epithionitriles are also formed, provided that the necessary enzymes are available. Epithionitriles have been shown to toxic to rat and cattle (Nishie and Daxenbichler, 1980; Collett et al., 2014) and CETB to rats (VanSteenhouse et al., 1999); however, to date the in planta role of nitriles and epithionitriles has been restricted to either herbivore/plant (Lambrix et al., 2001; Mumm et al., 2008) or fungus/plant interactions (Pedras and Hossain, 2011).

Here, the accessions which responded to $V$. longisporum colonization by a reduction in their leaf GLS content (Bur0 and Ler-0) also experienced a fall in their GLS breakdown product content (and vice versa for $\mathrm{Hi}-0$ and $\mathrm{Kn}-0$, Figure 4). The decrease in the leaf nitrile and epithionitrile content noted in Bur- 0 and Ler- 0 was consistent with the low level of ESP present in these accessions. The behavior of the roots was somewhat unexpected. Except for Hi-0, the content of most of the GLS breakdown products fell in response to the pathogen's colonization. In the two accessions classed as tolerant (Bur-0 and Ler-0), the production of both nitriles and ITCs was reduced, and in Bur- 0 , the recovery rate of GLS breakdown products dropped by $50 \%$ compared to the rate encountered in the noninfected root. GLS breakdown products have been shown to be antagonistic to the growth of Verticillium (Olivier et al., 1999; Down et al., 2004; Njoroge et al., 2011; Witzel et al., 2013), but the biochemical nature of this toxicity remains unclear. In the necrotrophic fungus Alternaria brassicicola, exposure to 2PropITC results in the induction of a number of genes associated with the oxidative burst and with cell cycle regulation (Sellam et al., 2007). When V. longisporum was presented with xylem sap obtained from oilseed rape, genes encoding both a number of heat-shock proteins and catalase peroxidase were up-regulated; knock-out mutants of these genes resulted in a $\mathrm{H}_{2} \mathrm{O}_{2}$ sensitive phenotype (Singh et al., 2012). Since xylem sap also contains GLS (Andersen et al., 2013) and myrosinase (Floerl et al., 2012), this induction may well be driven by the presence of GLS breakdown products. A working hypothesis for the reduced abundance of GLS breakdown products in infected $A$. thaliana roots is that these compounds interact with fungal targets such as glutathione or tubulin, thereby inhibiting the process of colonization. The product of the $V$. longisporum $\beta$-tubulin paralog could represent the relevant target (Inderbitzin et al., 2011). The tolerance of Bur0 , which experienced the most notable decrease in content of GLS breakdown products in the root, may be ascribable to this process.

\section{Conclusion}

Colonization of $A$. thaliana with $V$. longisporum influenced the tissue- and accession-specific accumulation of GLS and their respective breakdown products. Tolerant accessions might be more efficient in accumulating GLS at the infection site in the root. While our study did not identify a particular GLS or breakdown product associated with pathogen tolerance, further examinations should be extended to plants subjected to specific elicitors to accumulate specific GLS prior fungal inoculation (Wiesner et al., 2013a).

\section{Author Contributions}

Conceived and designed the experiments: KW, FH, RK, SR, MS, RG. Performed the experiments: KW, FH, RK. Analyzed the data: $\mathrm{KW}, \mathrm{FH}, \mathrm{RK}$. Contributed reagents/materials/analysis tools: SR, MS, RG. Wrote the paper: KW, FH, RK, SR, MS, RG.

\section{Acknowledgments}

The technical assistance of Sabine Breitkopf, Angelika Fandrey, Andrea Jankowsky, Annett Platalla, and Sieglinde Widiger is gratefully acknowledged. We thank Ralph Kissen for the gift of ESP antibody and Andreas von Tiedemann for the gift of $V$. longisporum isolate. This work was financially supported by the German Leibniz association (PAKT project 'Chemical Communication in the Rhizosphere,' SAW-2011IPB-3).

\section{Supplementary Material}

The Supplementary Material for this article can be found online at: http://journal.frontiersin.org/article/10.3389/fpls.2015.00508 


\section{References}

Agerbirk, N., De Vos, M., Kim, J. H., and Jander, G. (2009). Indole glucosinolate breakdown and its biological effects. Phytochem. Rev. 8, 101-120. doi: 10.1007/s11101-008-9098-0

Agerbirk, N., and Olsen, C. E. (2012). Glucosinolate structures in evolution. Phytochemistry 77, 16-45. doi: 10.1016/j.phytochem.2012.02.005

Aires, A., Dias, C. S. P., Carvalho, R., Oliveira, M. H., Monteiro, A. A., Simoes, M. V., et al. (2011). Correlations between disease severity, glucosinolate profiles and total phenolics and Xanthomonas campestris pv. campestris inoculation of different Brassicaceae. Sci. Hortic. 129, 503-510. doi: 10.1016/j.scienta.2011.04.009

Amme, S., Rutten, T., Melzer, M., Sonsmann, G., Vissers, J. P. C., Schlesier, B., et al. (2005). A proteome approach defines protective functions of tobacco leaf trichomes. Proteomics 5, 2508-2518. doi: 10.1002/pmic.200401274

Andersen, T. G., Nour-Eldin, H. H., Fuller, V. L., Olsen, C. E., Burow, M., and Halkier, B. A. (2013). Integration of biosynthesis and long-distance transport establish organ-specific glucosinolate profiles in vegetative Arabidopsis. Plant Cell 25, 3133-3145. doi: 10.1105/tpc.113.110890

Andersson, M. X., Nilsson, A. K., Johansson, O. N., Boztas, G., Adolfsson, L. E., Pinosa, F., et al. (2015). Involvement of the electrophilic isothiocyanate sulforaphane in Arabidopsis local defense responses. Plant Physiol. 167, 251261. doi: 10.1104/pp.114.251892

Atallah, Z. K., Hayes, R. J., and Subbarao, K. V. (2011). Fifteen years of Verticillium wilt of lettuce in America's salad bowl: a tale of immigration, subjugation, and abatement. Plant Dis. 95, 784-792. doi: 10.1094/PDIS-01-11-0075

Beekwilder, J., Van Leeuwen, W., Van Dam, N. M., Bertossi, M., Grandi, V., Mizzi, L., et al. (2008). The impact of the absence of aliphatic glucosinolates on insect herbivory in Arabidopsis. PLoS ONE 3:e2068. doi: 10.1371/journal.pone.0002068

Brader, G., Mikkelsen, M. D., Halkier, B. A., and Palva, E. T. (2006). Altering glucosinolate profiles modulates disease resistance in plants. Plant J. 46, 758767. doi: 10.1111/j.1365-313X.2006.02743.x

Burow, M., Losansky, A., Müller, R., Plock, A., Kliebenstein, D. J., and Wittstock, U. (2009). The genetic basis of constitutive and herbivore-induced ESP-independent nitrile formation in Arabidopsis. Plant Physiol. 149, 561-574. doi: 10.1104/pp.108.130732

Clay, N. K., Adio, A. M., Denoux, C., Jander, G., and Ausubel, F. M. (2009). Glucosinolate metabolites required for an Arabidopsis innate immune response. Science 323, 95-101. doi: 10.1126/science.1164627

Collett, M. G., Stegelmeier, B. L., and Tapper, B. A. (2014). Could nitrile derivatives of turnip (Brassica rapa) glucosinolates be hepato- or cholangiotoxic in cattle? J. Agric. Food Chem. 62, 7370-7375. doi: 10.1021/jf500526u

Daayf, F., Nicole, M., and Geiger, J. P. (1995). Differentiation of Verticillium dahliae populations on the basis of vegetative compatibility and pathogenicity on cotton. Eur. J. Plant Pathol. 101, 69-79. doi: 10.1007/BF01876095

Dervis, S., Yetisir, H., Y $\iota l d \imath$ r $\iota$ m, H., Tok, F., Kurt, S., and Karaca, F. (2009). Genetic and pathogenic characterization of Verticillium dahliae isolates from eggplant in Turkey. Phytoparasitica 37, 467-476. doi: 10.1007/s12600-009-0061-4

Down, G. J., Harris, D. C., and Murray, R. A. (2004). Destruction of Verticillium dahliae in soil following the addition of sulphur-containing volatile compounds potentially produced from Brassica tissues. Agroindustria 3, 293-294.

Doyle, J. J., and Doyle, J. L. (1990). Isolation of plant DNA from fresh tissue. Focus (Madison) 12, 13-15

Floerl, S., Majcherczyk, A., Possienke, M., Feussner, K., Tappe, H., Gatz, C., et al. (2012). Verticillium longisporum infection affects the leaf apoplastic proteome, metabolome, and cell wall properties in Arabidopsis thaliana. PLoS ONE 7:e31435. doi: 10.1371/journal.pone.0031435

Fradin, E. F., and Thomma, B. (2006). Physiology and molecular aspects of Verticillium wilt diseases caused by $V$. dahliae and V. albo-atrum. Mol. Plant Pathol. 7, 71-86. doi: 10.1111/j.1364-3703.2006.00323.x

Gebhardt, C., Ritter, E., Debener, T., Schachtschabel, U., Walkemeier, B., Uhrig, H., et al. (1989). RFLP analysis and linkage mapping in Solanum tuberosum. Theor. Appl. Genet. 78, 65-75. doi: 10.1007/BF00299755

Gibeaut, D. M., Hulett, J., Cramer, G. R., and Seemann, J. R. (1997). Maximal biomass of Arabidopsis thaliana using a simple, low-maintenance hydroponic method and favorable environmental conditions. Plant Physiol. 115, 317-319. doi: 10.1104/pp.115.2.317
Häffner, E., Karlovsky, P., and Diederichsen, E. (2010). Genetic and environmental control of the Verticillium syndrome in Arabidopsis thaliana. BMC Plant Biol. 10:235. doi: 10.1186/1471-2229-10-235

Häffner, E., Karlovsky, P., Splivallo, R., Traczewska, A., and Diederichsen, E. (2014). ERECTA, salicylic acid, abscisic acid, and jasmonic acid modulate quantitative disease resistance of Arabidopsis thaliana to Verticillium longisporum. BMC Plant Biol. 14:85. doi: 10.1186/1471-2229-14-85

Hanschen, F. S., Lamy, E., Schreiner, M., and Rohn, S. (2014). Reactivity and stability of glucosinolates and their breakdown products in foods. Angew. Chem. Int. Ed. Engl. 53, 11430-11450. doi: 10.1002/anie.201 402639

Hara, M., Harazaki, A., and Tabata, K. (2013). Administration of isothiocyanates enhances heat tolerance in Arabidopsis thaliana. Plant Growth Regul. 69, 71-77. doi: 10.1007/s10725-012-9748-5

Hara, M., Yatsuzuka, Y., Tabata, K., and Kuboi, T. (2010). Exogenously applied isothiocyanates enhance glutathione S-transferase expression in Arabidopsis but act as herbicides at higher concentrations. J. Plant Physiol. 167, 643-649. doi: 10.1016/j.jplph.2009.11.006

Hossain, M. S., Ye, W. X., Hossain, M. A., Okuma, E., Uraji, M., Nakamura, Y., et al. (2013). Glucosinolate degradation products, isothiocyanates, nitriles, and thiocyanates, induce stomatal closure accompanied by peroxidase-mediated reactive oxygen species production in Arabidopsis thaliana. Biosci. Biotechnol. Biochem. 77, 977-983. doi: 10.1271/bbb.120928

Inderbitzin, P., Davis, R. M., Bostock, R. M., and Subbarao, K. V. (2011). The ascomycete Verticillium longisporum is a hybrid and a plant pathogen with an expanded host range. PLoS ONE 6:e18260. doi: 10.1371/journal.pone.0018260

Iven, T., Konig, S., Singh, S., Braus-Stromeyer, S. A., Bischoff, M., Tietze, L. F., et al. (2012). Transcriptional activation and production of tryptophan-derived secondary metabolites in Arabidopsis roots contributes to the defense against the fungal vascular pathogen Verticillium longisporum. Mol. Plant. 5, 13891402. doi: $10.1093 / \mathrm{mp} / \mathrm{sss} 044$

Jimenez-Diaz, R. M., Cirulli, M., Bubici, G., Jimenez-Gasco, M. D., Antoniou, P. P., and Tjamos, E. C. (2012). Verticillium wilt, a major threat to olive production: current status and future prospects for its management. Plant Dis. 96, 304-329. doi: 10.1094/PDIS-06-11-0496

Johansson, A., Staal, J., and Dixelius, C. (2006). Early responses in the ArabidopsisVerticillium longisporum pathosystem are dependent on NDR1, JA- and ETassociated signals via cytosolic NPR1 and RFO1. Mol. Plant Microbe Interact. 19, 958-969. doi: 10.1094/MPMI-19-0958

Khokon, M. A. R., Jahan, M. S., Rahman, T., Hossain, M. A., Muroyama, D., Minami, I., et al. (2011). Allyl isothiocyanate (AITC) induces stomatal closure in Arabidopsis. Plant Cell Environ. 34, 1900-1906. doi: 10.1111/j.13653040.2011.02385.x

Kissen, R., and Bones, A. M. (2009). Nitrile-specifier proteins involved in glucosinolate hydrolysis in Arabidopsis thaliana. J. Biol. Chem. 284, 1205712070. doi: 10.1074/jbc.M807500200

Kissen, R., Hyldbakk, E., Wang, C. W. V., Sormo, C. G., Rossiter, J. T., and Bones, A. M. (2012). Ecotype dependent expression and alternative splicing of epithiospecifier protein (ESP) in Arabidopsis thaliana. Plant Mol. Biol. 78, 361-375. doi: 10.1007/s11103-011-9869-7

Kjaer, A. (1963). Mass spectra of isothiocyanates. Acta Chem. Scand. 17, 2143-2154. doi: 10.3891/acta.chem.scand.17-2143

König, S., Feussner, K., Kaever, A., Landesfeind, M., Thurow, C., Karlovsky, P., et al. (2014). Soluble phenylpropanoids are involved in the defense response of Arabidopsis against Verticillium longisporum. New Phytol. 202, 823-837. doi: 10.1111/nph.12709

Lambrix, V., Reichelt, M., Mitchell-Olds, T., Kliebenstein, D. J., and Gershenzon, J. (2001). The Arabidopsis epithiospecifier protein promotes the hydrolysis of glucosinolates to nitriles and influences Trichoplusia ni herbivory. Plant Cell 13, 2793-2807. doi: 10.1105/tpc.13.12.2793

Li, K. N., Rouse, D. I., Eyestone, E. J., and German, T. L. (1999). The generation of specific DNA primers using random amplified polymorphic DNA and its application to Verticillium dahliae. Mycol. Res. 103, 1361-1368. doi: 10.1017/S0953756299008357

Madsen, S. R., Olsen, C. E., Nour-Eldin, H. H., and Halkier, B. A. (2014). Elucidating the role of transport processes in leaf glucosinolate distribution. Plant Physiol. 166, 1450-1462. doi: 10.1104/pp.114. 246249 
Markovich, O., Kafle, D., Elbaz, M., Malitsky, S., Aharoni, A., Schwarzkopf, A., et al. (2013). Arabidopsis thaliana plants with different levels of aliphatic- and indolyl-glucosinolates affect host selection and performance of Bemisia tabaci. J. Chem. Ecol. 39, 1361-1372. doi: 10.1007/s10886-013-0358-0

Martinez-Ballesta, M. D., Moreno, D. A., and Carvajal, M. (2013). The physiological importance of glucosinolates on plant response to abiotic stress in Brassica. Int. J. Mol. Sci. 14, 11607-11625. doi: 10.3390/ijms 140611607

Matusheski, N. V., and Jeffery, E. H. (2001). Comparison of the bioactivity of two glucoraphanin hydrolysis products found in broccoli, sulforaphane and sulforaphane nitrile. J. Agric. Food Chem. 49, 5743-5749. doi: 10.1021/jf010809a

Mewis, I., Khan, M. A. M., Glawischnig, E., Schreiner, M., and Ulrichs, C. (2012a). Water stress and aphid feeding differentially influence metabolite composition in Arabidopsis thaliana (L.). PLoS ONE 7:e48661. doi: 10.1371/journal.pone.0048661

Mewis, I., Schreiner, M., Nguyen, C. N., Krumbein, A., Ulrichs, C., Lohse, M., et al. (2012b). UV-B irradiation changes specifically the secondary metabolite profile in broccoli sprouts: induced signaling overlaps with defense response to biotic stressors. Plant Cell Physiol. 53, 1546-1560. doi: 10.1093/pcp/ pcs096

Mi, L., Xiao, Z., Hood, B. L., Dakshanamurthy, S., Wang, X., Govind, S., et al. (2008). Covalent binding to tubulin by isothiocyanates: a mechanism of cell growth arrest and apoptosis. J. Biol. Chem. 283, 22136-22146. doi: 10.1074/jbc.M802330200

Moore, B., Andrew, R. L., Kulheim, C., and Foley, W. J. (2014). Explaining intraspecific diversity in plant secondary metabolites in an ecological context. New Phytol. 201, 733-750. doi: 10.1111/nph.12526

Mumm, R., Burow, M., Bukovinszkine'kiss, G., Kazantzidou, E., Wittstock, U., Dicke, M., et al. (2008). Formation of simple nitriles upon glucosinolate hydrolysis affects direct and indirect defense against the specialist herbivore, Pieris rapae. J. Chem. Ecol. 34, 1311-1321. doi: 10.1007/s10886008-9534-z

Nishie, K., and Daxenbichler, M. E. (1980). Toxicology of glucosinolates, related-compounds (nitriles, R-goitrin, isothiocyanates) and vitamin $\mathrm{U}$ found in cruciferae. Food Cosmet. Toxicol. 18, 159-172. doi: 10.1016/00156264(80)90070-X

Njoroge, S. M. C., Vallad, G. E., Park, S. Y., Kang, S., Koike, S. T., Bolda, M., et al. (2011). Phenological and phytochemical changes correlate with differential interactions of Verticillium dahliae with broccoli and cauliflower. Phytopathology 101, 523-534. doi: 10.1094/PHYTO-08-10-0219

Obermeier, C., Hossain, M. A., Snowdon, R., Knufer, J., Von Tiedemann, A., and Friedt, W. (2013). Genetic analysis of phenylpropanoid metabolites associated with resistance against Verticillium longisporum in Brassica napus. Mol. Breed. 31, 347-361. doi: 10.1007/s11032-012-9794-8

Olivier, C., Vaughn, S. F., Mizubuti, E. S. G., and Loria, R. (1999). Variation in allyl isothiocyanate production within Brassica species and correlation with fungicidal activity. J. Chem. Ecol. 25, 2687-2701. doi: 10.1023/A:1020895306588

Pantelides, I. S., Tjamos, S. E., and Paplomatas, E. J. (2010). Ethylene perception via ETR1 is required in Arabidopsis infection by Verticillium dahliae. Mol. Plant Pathol. 11, 191-202. doi: 10.1111/j.1364-3703.2009.00592.x

Pedras, M. S. C., and Hossain, S. (2011). Interaction of cruciferous phytoanticipins with plant fungal pathogens: indole glucosinolates are not metabolized but the corresponding desulfo-derivatives and nitriles are. Phytochemistry 72, 23082316. doi: 10.1016/j.phytochem.2011.08.018

Piekarska, A., Koiodziejski, D., Pilipczuk, T., Bodnar, M., Konieczka, P., Kusznierewicz, B., et al. (2014). The influence of selenium addition during germination of Brassica seeds on health-promoting potential of sprouts. Int. J. Food Sci. Nutr. 65, 692-702. doi: 10.3109/09637486.2014.917148

Reusche, M., Thole, K., Janz, D., Truskina, J., Rindfleisch, S., Drubert, C., et al. (2012). Verticillium infection triggers VASCULAR-RELATED NAC DOMAIN7-dependent de novo xylem formation and enhances drought tolerance in Arabidopsis. Plant Cell 24, 3823-3837. doi: 10.1105/tpc.112.103374

Rohr, F., Ulrichs, C., Schreiner, M., Zrenner, R., and Mewis, I. (2012). Responses of Arabidopsis thaliana plant lines differing in hydroxylation of aliphatic glucosinolate side chains to feeding of a generalist and specialist caterpillar. Plant Physiol. Biochem. 55, 52-59. doi: 10.1016/j.plaphy.2012.03.005

Roos, J., Bejai, S., Oide, S., and Dixelius, C. (2014). RabGAP22 is required for defense to the vascular pathogen Verticillium longisporum and contributes to stomata immunity. PLoS ONE 9:e88187. doi: 10.1371/journal.pone.0088187
Saeed, A. I., Sharov, V., White, J., Li, J., Liang, W., Bhagabati, N., et al. (2003). TM4: a free, open-source system for microarray data management and analysis. Biotechniques 34, 374-378.

Sellam, A., Dongo, A., Guillemette, T., Hudhomme, P., and Simoneau, P. (2007). Transcriptional responses to exposure to the brassicaceous defence metabolites camalexin and allyl-isothiocyanate in the necrotrophic fungus Alternaria brassicicola. Mol. Plant Pathol. 8, 195-208. doi: 10.1111/j.13643703.2007.00387.x

Shofran, B. G., Purrington, S. T., Breidt, F., and Fleming, H. P. (1998). Antimicrobial properties of sinigrin and its hydrolysis products. J. Food Sci. 63, 621-624. doi: 10.1111/j.1365-2621.1998.tb 15798.x

Singh, S., Braus-Stromeyer, S. A., Timpner, C., Valerius, O., Von Tiedemann, A., Karlovsky, P., et al. (2012). The plant host Brassica napus induces in the pathogen Verticillium longisporum the expression of functional catalase peroxidase which is required for the late phase of disease. Mol. Plant Microbe Interact. 25, 569-581. doi: 10.1094/MPMI-08-11-0217

Spencer, G. F., and Daxenbichler, M. E. (1980). Gas chromatography-mass spectrometry of nitriles, isothiocyanates and oxazolidinethiones derived from cruciferous glucosinolates. J. Sci. Food Agric. 31, 359-367. doi: $10.1002 /$ jsfa. 2740310406

Stotz, H. U., Sawada, Y., Shimada, Y., Hirai, M. Y., Sasaki, E., Krischke, M., et al. (2011). Role of camalexin, indole glucosinolates, and side chain modification of glucosinolate-derived isothiocyanates in defense of Arabidopsis against Sclerotinia sclerotiorum. Plant J. 67, 81-93. doi: 10.1111/j.1365313X.2011.04578.x

Textor, S., and Gershenzon, J. (2009). Herbivore induction of the glucosinolateâ€"myrosinase defense system: major trends, biochemical bases and ecological significance. Phytochem. Rev. 8, 149-170. doi: $10.1007 /$ s11101-008-9117-1

Tinker, N. A., Fortin, M. G., and Mather, D. E. (1993). Random amplified polymorphic DNA and pedigree relationships in spring barley. Theor. Appl. Genet. 85, 976-984. doi: 10.1007/BF00215037

Tischner, R., Koltermann, M., Hesse, H., and Plath, M. (2010). Early responses of Arabidopsis thaliana to infection by Verticillium longisporum. Physiol. Mol. Plant Pathol. 74, 419-427. doi: 10.1016/j.pmpp.2010. 06.004

Tsror, L., Hazanovsky, M., Mordechi-Lebiush, S., and Sivan, S. (2001). Aggressiveness of Verticillium dahliae isolates from different vegetative compatibility groups to potato and tomato. Plant Pathol. 50, 477-482. doi: 10.1046/j.1365-3059.2001.00587.x

van Ommen Kloeke, A. E. E., Van Gestel, C. M., Styrishave, B., Hansen, M., Ellers, J., and Roelofs, D. (2012). Molecular and life-history effects of a natural toxin on herbivorous and non-target soil arthropods. Ecotoxicology 21, 1084-1093. doi: 10.1007/s10646-012-0861-z

VanSteenhouse, J. L., Prescott, J. S., and Swenson, D. H. (1999). Protection from 1-cyano-3,4-epithiobutane nephrotoxicity by aminooxyacetic acid and effect on xenobiotic-metabolizing enzymes in male Fischer 344 rats. J. Appl. Toxicol. 19, 237-249. doi: 10.1002/(SICI)1099-1263(199907/08)19:4<237::AIDJAT569>3.0.CO;2-7

Veronese, P., Narasimhan, M. L., Stevenson, R. A., Zhu, J. K., Weller, S. C., Subbarao, K. V., et al. (2003). Identification of a locus controlling Verticillium disease symptom response in Arabidopsis thaliana. Plant J. 35, 574-587. doi: 10.1046/j.1365-313X.2003.01830.x

Wiesner, M., Hanschen, F. S., Schreiner, M., Glatt, H., and Zrenner, R. (2013a). Induced production of 1-methoxy-indol-3-ylmethyl glucosinolate by jasmonic acid and methyl jasmonate in sprouts and leaves of pak choi (Brassica rapa ssp chinensis). Int. J. Mol. Sci. 14, 14996-15016. doi: 10.3390/ijms140 714996

Wiesner, M., Zrenner, R., Krumbein, A., Glatt, H., and Schreiner, M. (2013b). Genotypic variation of the glucosinolate profile in pak choi (Brassica rapa ssp. chinensis). J. Agric. Food Chem. 61, 1943-1953. doi: 10.1021/jf3 03970k

Wittstock, U., and Burow, M. (2010). Glucosinolate breakdown in Arabidopsis: mechanism, regulation and biological significance. Arabidopsis Book 8, e0134. doi: 10.1199/tab.0134

Wittstock, U., Kliebenstein, D. J., Lambrix, V., Reichelt, M., and Gershenzon, J. (2003). "Chapter five Glucosinolate hydrolysis and 
its impact on generalist and specialist insect herbivores," in Recent Advances in Phytochemistry, ed. T. R. John (Philadelphia, PA: Elsevier), 101-125.

Witzel, K., Hanschen, F. S., Schreiner, M., Krumbein, A., Ruppel, S., and Grosch, R. (2013). Verticillium suppression is associated with the glucosinolate composition of Arabidopsis thaliana leaves. PLoS ONE 8:e71877. doi: 10.1371/journal.pone.0071877

Zeise, K., and von Tiedemann, A. (2002). Host specialization among vegetative compatibility groups of Verticillium dahliae in relation to Verticillium longisporum. J. Phytopathol. 150, 112-119. doi: 10.1046/j.1439-0434.2002.00730.x

Zhang, Z., Ober, J. A., and Kliebenstein, D. J. (2006). The gene controlling the quantitative trait locus EPITHIOSPECIFIER MODIFIER1 alters glucosinolate hydrolysis and insect resistance in Arabidopsis. Plant Cell 18, 1524-1536. doi: $10.1105 /$ tpc. 105.039602
Zhou, L., Hu, Q., Johansson, A., and Dixelius, C. (2006). Verticillium longisporum and $V$. dahliae: infection and disease in Brassica napus. Plant Pathol. 55, 137-144. doi: 10.1111/j.1365-3059.2005.01311.x

Conflict of Interest Statement: The authors declare that the research was conducted in the absence of any commercial or financial relationships that could be construed as a potential conflict of interest.

Copyright (๑) 2015 Witzel, Hanschen, Klopsch, Ruppel, Schreiner and Grosch. This is an open-access article distributed under the terms of the Creative Commons Attribution License (CC BY). The use, distribution or reproduction in other forums is permitted, provided the original author(s) or licensor are credited and that the original publication in this journal is cited, in accordance with accepted academic practice. No use, distribution or reproduction is permitted which does not comply with these terms. 